\title{
Peritonitis tuberculosa: ¿una enfermedad del Tercer Mundo?
}

\author{
R. LÓPEZ RODRÍGUEZ, J. CAMPOS FRANCO, F. L. LADO LADO, M. R. ALENDE \\ SIXTO, A. GONZÁLEZ QUINTELA
}

Departamento de Medicina. Unidad Médica de Alta Precoz. Complejo Hospitalario

Universitario de Santiago de Compostela. Santiago de Compostela. A Coruña

TUBERCULOUS PERITONITIS: A THIRD WORLD'S DISEASE?

\begin{abstract}
RESUMEN
La tuberculosis peritoneal todavía causa una importante morbi-mortalidad. La evolución clínica de la enfermedad depende de la rapidez en el diagnóstico y en el inicio del tratamiento. Hemos revisado cinco casos de tuberculosis peritoneal diagnosticados en una unidad médica de corta estancia durante el período de un año. Todos los pacientes respondieron favorablemente al tratamiento. Se discute el manejo clínico de la tuberculosis peritoneal y se revisa el papel actual de la tomografía computerizada y otras técnicas en el diagnóstico de esta enfermedad.
\end{abstract}

PALABRAS CLAVE: Tuberculosis. Peritonitis. Ascitis.

\begin{abstract}
Tuberculous peritonitis has a high morbidity and mortality. The clinical outcome of tuberculous peritonitis depends on an early diagnosis and treatment. We review five cases of tuberculous peritonitis diagnosed in a short stay medical unit over a one-year period. All patients were succesfully treated with antituberculous drugs. The clinical management of this disease is described and current views regarding the value of computed tomography and other diagnostic techniques are discussed.
\end{abstract}

KEY WORDS: Tuberculosis. Peritonitis. Ascites.

López Rodríguez R, Campos Franco J, Lado Lado FL, Alende Sixto MR, González Quintela A. Peritonitis tuberculosa: ¿una enfermedad del Tercer Mundo? An Med Interna (Madrid) 2004; 21: 331-333.

\section{INTRODUCCIÓN}

La tuberculosis continúa siendo, hoy en día, un problema sanitario en España con una incidencia media de 40 casos $\mathrm{x}$ 100.000 habitantes/año (1). Dentro del espectro clínico de la tuberculosis, la afectación peritoneal es una forma de presentación poco frecuente. Representa, en las distintas series publicadas, del 2 al 7,3\% de los nuevos casos de tuberculosis en España (2-5) y la tercera causa de ascitis después de la cirrosis y las neoplasias (6), mientras que en otros países desarrollados representa el $0,6 \%$ de los nuevos casos de tuberculosis y la sexta forma de presentación extrapulmonar (7). Esto supone una incidencia de 4 a 8 veces superior a la observada en otros países industrializados. Esta forma de tuberculosis está aumentando en los países occidentales, asociada a la inmigración y a la infección por el VIH $(8,9)$, por lo que debe tenerse presente en el diagnóstico diferencial de cuadros que cursen con fiebre y ascitis, especialmente en áreas donde la tuberculosis es endémica, como es nuestro caso, y en aquellas zonas sometidas a movimientos migratorios.

Presentamos cinco casos de peritonitis tuberculosa diagnosticados en nuestra Unidad en un período de 12 meses (junio 2002- junio 2003), lo que supone un marcado aumento de su incidencia, y se analizan sus características epidemiológicas, manifestaciones clínicas e implicaciones diagnósticas

\section{CASOS APORTADOS}

Durante un periodo de 12 meses (junio 2002-junio 2003) se diagnosticaron cinco casos de peritonitis tuberculosa en la Unidad Médica de Alta Precoz, adscrita al Servicio de Medicina Interna del Hospital Clínico Universitario de Santiago de Compostela. Tres eran mujeres y dos eran varones, con una edad media de 31,8 años (rango: 20-49 años). En el 60\% existía un antecedente de exposición reciente a $M$. tuberculosis y mantoux negativo en ese momento. La PPD fue positiva en tres casos, de los cuales dos habían estado en contacto con un portador de tuberculosis pulmonar. Ninguno era emigrante, todos eran VIH negativos y una paciente era portadora de una hepatopatía de probable origen enólico. Las manifestaciones clínicas fueron: fiebre $(100 \%)$, dolor abdominal $(100 \%)$, ascitis $(100 \%)$, distensión abdominal (60\%), síndrome general $(40 \%)$, diarrea $(40 \%)$, cefalea $(40 \%)$. Las pruebas de laboratorio se expone en la tabla I.

A todos los pacientes se les realizaron radiografías de tórax al ingreso, (que demostró alteraciones en un caso en forma de derrame

Trabajo aceptado: 18 de febrero de 2004

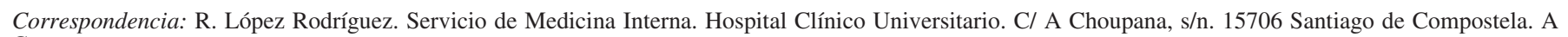
Coruña 
TABLA I

\begin{tabular}{lccccc}
\multicolumn{7}{c}{ RESULTADOS DE LABORATORIO } \\
\hline & Caso 1 & Caso 2 & Caso 3 & Caso 4 & Caso 5 \\
\hline Leucocitos & 3.580 & 3.950 & 10.360 & 7.010 & 9.780 \\
Linfocitos (\%) & 20 & 11 & 9,6 & 9 & 10 \\
Cayados (\%) & 7 & 25 & - & 5 & - \\
Hb (g/dL) & 12 & 10,4 & 11,5 & 11,4 & 10,7 \\
T. quick (\%) & 75 & 67 & 73 & 67 & 66 \\
VSC (mm 1 ${ }^{\text {a }}$ h) & 61 & 59 & 51 & 0 & 114 \\
\hline
\end{tabular}

pleural y signos de tuberculosis residual) y durante su estancia (objetivándose en dos casos el desarrollo de afectación pleural). En todos los casos se demostró, mediante ecografía abdominal, la presencia de líquido libre y en cuatro casos se realizó una tomografía axial computerizada (TAC) abdominal, que presentó en todos ellos hallazgos compatibles con peritonitis tuberculosa.

Se estudió el líquido ascítico en la totalidad de los pacientes, realizándose recuento celular, estudio bioquímico, ADA (adenosín deaminasa), Ca 125 (sólo en tres casos), estudio microbiológico y anatomopatológico, cuyos resultados se exponen en la tabla II.

El tiempo medio transcurrido desde el ingreso en el hospital hasta el diagnóstico fue de 4,6 días (rango: 3-7 días). Se trataron todos los pacientes con triple terapia, isoniacida, rifampicina y pirazinamida, con defervescencia de la fiebre en 2-3 días y buena evolución clínica.

TABLA ॥

\begin{tabular}{|c|c|c|c|c|c|}
\hline \multicolumn{6}{|c|}{ CARACTERÍSTICAS DEL LÍQUIDO ASCÍTICO } \\
\hline & Caso 1 & Caso 2 & Caso 3 & Caso 4 & Caso 5 \\
\hline $\mathrm{Cel} / \mathrm{mm}$ & 3.900 & 2.300 & 450 & 2.100 & 9.490 \\
\hline Linfocitos (\%) & 85 & 60 & 76 & 50 & 65 \\
\hline Proteínas $\mathrm{g} / \mathrm{dL}$ & & & & & \\
\hline $\begin{array}{l}<3 \mathrm{~g} / \mathrm{dL} \text { ) } \\
\text { jlucosamg/dL }\end{array}$ & 4,9 & 5 & 3 & 5,1 & 5,5 \\
\hline $\begin{array}{l}\text { (> } 60 \text { mg/dL) } \\
\text { LDH UI/L }\end{array}$ & 77 & 35 & 82 & 106 & \\
\hline $\begin{array}{l}(<200 \mathrm{UI} / \mathrm{L}) \\
\mathrm{ADA} U / \mathrm{L}\end{array}$ & 634 & 1237 & 269 & 622 & 1.398 \\
\hline (<40 U/L) & 114 & 111 & 50 & 150 & 98 \\
\hline CA-125U/L & 1.712 & 1.115 & & & 2.956 \\
\hline AAR & Negativo & Negativo & Negativo & Negativo & Negativo \\
\hline CR & Negativo & Negativo & & Negativo & Negativo \\
\hline $\begin{array}{l}\text { Lowenstein } \\
\text { Anatomía }\end{array}$ & Positivo & Positivo & Negativo & Negativo & Negativo \\
\hline & Linfocitosis & Linfocitosis & Linfocitosis & Linfocitosis & Linfocitosis \\
\hline
\end{tabular}

\section{DISCUSIÓN}

La tuberculosis peritoneal es una forma rara de presentación de la enfermedad, que en España representa del 2 al 7,3\% de los nuevos casos (2-5). Se produce por la siembra desde los ganglios linfáticos, el intestino, las trompas de Falopio, o menos frecuentemente, como consecuencia de la diseminación hematógena o linfática desde un foco primario distante $(2,4-6,10)$. No es infrecuente que se afecten otras serosas $(3,10,11)$; en uno de nuestros casos existía derrame pleural en el momento del ingre- so y otros dos lo desarrollaron a lo largo del mismo. Probablemente sea debido al paso de ascitis a la cavidad pleural a través del diafragma, como ocurre en el hidrotórax hepático. Se considera que la enfermedad se presenta, en los países industrializados, por encima de los 40 años $(2,3,10)$; mientras que en otros menos avanzados o con inmigrantes se produce entre los $20 \mathrm{y}$ los 30 años $(9,10)$. Tres de nuestros pacientes tenían menos de 22 años, ninguno de los cuales era emigrante, y los otros dos contaban con más de 47 años. Este hallazgo, sin duda alguna, debe de relacionarse íntimamente con la incidencia de la tuberculosis en España, donde es más fácil entrar en contacto con M. tuberculosis que en otros países occidentales.

En los países desarrollados es infrecuente la exposición a la tuberculosis y se consideran factores de riesgo la infección por el VIH, la adicción a drogas por vía parenteral, el alcoholismo, la cirrosis hepática, la diabetes, el cáncer y el tratamiento esteroideo (5,8-12). En nuestra serie el $60 \%$ presentaban un contacto reciente con la tuberculosis. Sólo un paciente presentaba como factor de riesgo etilismo y una hepatopatía de probable origen enólico.

Está descrito en la literatura que la prueba de la tuberculina puede ser negativa en la peritonitis tuberculosa hasta en el 70\% de los casos, siendo junto con la miliar, las dos formas de tuberculosis extrapulmonar donde el mantoux es con más frecuencia negativo y se ha puesto en relación con la inmunodepresión que padecen estos pacientes $(3,5,10,11)$. Nosotros le realizamos la prueba a todos los pacientes y resultó positiva en tres casos.

Los síntomas de la peritonitis tuberculosa son generalmente inespecíficos, aunque generalmente domina el cuadro clínico el dolor abdominal, la ascitis y la fiebre $(2-4,6,9-11,13)$. Todos nuestros pacientes presentaban fiebre, dolor abdominal y ascitis demostrada mediante un ecógrafo que disponemos en la Unidad y que en algún caso, debido a la escasa cantidad de líquido ascítico, se utilizó para marcar la zona donde sería más rentable realizar la paracentesis.

Las pruebas de laboratorio tampoco aportan mucho al diagnóstico $(5,10,11)$. Destacamos que todos los casos presentaron linfopenia, anemia, VSG $>50 \mathrm{~mm}$ a la primera hora y descenso ligero del tiempo de Quick.

El líquido ascítico suele ser un exudado de predominio linfocitario $(5,10)$, aunque en ocasiones puede presentar características de trasudado, sobre todo en pacientes cirróticos $(4,14-16)$. Los valores de LDH $>90 \mathrm{UI} / 1$ presentan una alta sensibilidad (90\%) y una baja especificidad para el diagnóstico de tuberculosis peritoneal (16), en nuestra serie estaba por encima de esos valores en todos los pacientes. El Ca-125 es un marcador tumoral del carcinoma de ovario que diversos estudios han demostrado que se encuentra elevado, en el suero y el líquido ascítico, de los pacientes con peritonitis tuberculosa, llegándose a establecer que podría ser utilizado para el diagnóstico y, su normalización, como marcador de respuesta al tratamiento tuberculostático (1720). En la mayoría de los casos se tratan de mujeres en la edad media de la vida; determinamos el Ca-125 en el líquido ascítico de tres pacientes, dos hombres y una mujer, y en todos los casos presentaron valores por encima de $1000 \mathrm{U} / \mathrm{mL}$. La determinación de la actividad de la ADA en el líquido ascítico ha demostrado ser de gran utilidad en el diagnóstico de la peritonitis tuberculosa, con una sensibilidad del $94 \%-100 \%$ y una especificidad del 92\%- 100\%, para valores superiores a $30 \mathrm{U} / \mathrm{L}$ $(13,15,21,22)$. De tal manera que valores elevados de ADA pueden considerarse diagnósticos y permitirnos evitar la realización 
de pruebas invasivas y potencialmente peligrosas como la laparoscopia $(9,13,23,24)$. Todos nuestros pacientes presentaron valores de ADA por encima del punto de corte considerado como diagnóstico. Es un hecho constatado la baja rentabilidad del BAAR, PCR y cultivo del líquido ascítico para el aislamiento del M. tuberculosis $(5,9,11,13,16,25)$; en nuestra serie, el cultivo sólo fue positivo en el $40 \%$ de los casos, mientras que el BAAR y la PCR fueron negativas.

En los últimos tiempos se ha puesto de manifiesto la utilidad de las pruebas de imagen, TAC y resonancia nuclear magnética, en el diagnóstico de múltiples patologías. La peritonitis tuberculosa no es una excepción y la presencia de ascitis, libre o loculada, con un valor de alta atenuación (20-45 HU), adenopatías intraabdominales y engrosamiento del peritoneo parietal con afectación del omento y mesenterio son altamente sugestivas de esta enfermedad (26-28). Su utilización, en combinación con los otros parámetros, puede evitar la realización de laparoscopias $(26,28)$, ya que la mayor parte de las ocasiones estas se realizan más para descartar procesos neoformativos que para diagnosti- car la peritonitis tuberculosa. El principal problema que presenta es establecer el diagnóstico diferencial con la carcinomatosis peritoneal; difícil de realizar, en ocasiones, sólo a través de la imagen. En nuestra serie se realizó estudio anatomopatológico de líquido ascítico en todos los casos, objetivándose la presencia de linfocitosis y la ausencia de células atípicas.

En todos los casos se objetivó una buena respuesta, en poco tiempo, al tratamiento tuberculostático, con defervescencia de la fiebre y desaparición progresiva de la ascitis.

Como conclusión, la tuberculosis peritoneal debe considerarse en el diagnóstico diferencial de los cuadros de ascitis y fiebre, especialmente en áreas endémicas, donde los datos radiológicos, el estudio del líquido ascítico y la respuesta al tratamiento tuberculostático permiten alcanzar el diagnóstico de un forma rápida y segura. Creemos que deberían reservarse las pruebas diagnósticas invasivas, lapatomía y laparoscopia, no exentas de complicaciones, para aquellos casos en los que no se alcance un diagnóstico o existan serias dudas de carcinomatosis peritoneal.

\section{Bibliografía}

1. Rodrigo T, Gaylá JA, Galdós-Tanguis H, Jansa JM, Brugal T, García de Olalla P. Evaluación de los programas de control de tuberculosis en las Comunidades Autónomas de España. Med Clin (Barc) 1999; 113: 604-7.

2. Maradona Hidalgo JA, Álvarez Álvarez C, Fernández Rippe ML, Suárez García E. Perspectiva actual de la tuberculosis peritoneal. Estudio de 26 pacientes. Rev Clin Esp 1983; 170: 133-6.

3. Zulaica D, Clave E, Torrado J, González MV, Etxebarría H, Damiano A. Tuberculosis peritoneal. Incidencia en una residencia sanitaria durante un período de siete años. Rev Clin Esp 1984; 172: 95-6.

4. Carballo Fernández C, Cutrín Prieto C, Murias Taboada E, Lado Lado F, Otero Echart M, Rodríguez López I, et al. Peritonitis tuberculosa. An Med Interna (Madrid) 1986; 3: 337-9.

5. Bilbao Garay J, Martínez L de Letona J, Carreño MC, Pérez Maestu R. Tuberculosis abdominal en la época actual. Revisión de 46 casos. Rev Clin Esp 1992; 191: 19-24.

6. Salva JA, Puig M, Jornet J, Broggi MA, Buera M, Sueiras A. Peritonitis tuberculosa. Presentación de 6 casos. Rev Clin Esp 1980; 156: 147-52.

7. Mehta JB, Dutt A, Harvill L, Mathews KM. Epidemiology of extrapulmonary tuberculosis. A comparative analysis with pre-AIDS era. Chest 1991; 99: 1134-8.

8. Raviglione MC, Snider DE, Kochi AQ. Global epidemiology of tuberculosis: morbidity and mortality of a world epidemic. JAMA 1995; 273 : 220-6.

9. Thoreau N, Fain O, Babinet P, Lortholary O, Robineau M, Valeyre D, et al. Tuberculose péritonéale: 27 cas dans la banlieve nord-est de Paris. Int J Tuberc Lung Dis 2002; 6: 253-8.

10. González Anglada MI, Barbado Hernández FJ, Cano Ruiz A, Cano López JN, Gamallo C, Sendino A, et al. Tuberculosis peritoneal. Características de una no rara localización tuberculosa. Rev Clin Esp 1992; 190: 393-7.

11. Lado Lado FL, Cabana González B, Ferreiro Regueiro MJ, Cabarcos Ortiz de Barrón A, Donado Budiño E. Peritonitis tuberculosa. Aportación de tres casos. An Med Interna (Madrid) 2002; 19: 296-8.

12. Hulnick DH, Megibow AJ, Naidcyh DP, Hilton S, Cho RC, Balthazar EJ. Abdominal tuberculosis: CT evaluation. Radiology 1985; 157: 199-204.

13. Marshall JB. Tuberculosis of the gastrointestinal tract and peritoneum. Am J Gastroenterol 1993; 88: 989-9.

14. Torralba M, Sáez JA, Solera J, Puras A. Trasudado peritoneal y ADA bajo en la peritonitis tuberculosa. Rev Clin Esp 1992; 191: 340-1.
15. Giménez Roca A, Xiol X, Castellote J, Sánchez M, Iglesias C, Ramón $\mathrm{JM}$, et al. Valor del ADA en la tuberculosis peritoneal. Rev Esp Enf Digest 1992; 82: 32-4.

16. Shakil AO, Korula J, Kanel GC, Murray NGB, Reynolds TB. Diagnostic features of tuberculous peritonitis in absence and presence of chronic liver disease: a case control study. Am J Med 1996; 100: 179-85.

17. Simsek H, Savas MC, Kadayifci A, Tatar G. Elevated serum CA-125 concentration in patients with tuberculous pèritonitis: a case control study. Am J Gastroenterol 1997; 92: 1.174-6.

18. Saavedra López J, Cabello Melendo P, De Gispert Servitge A, et al. Peritoneal tuberculosis with elevated CA-125 in serum and ascitis fluid. Gastroenterol Hepatol 1999; 22: 433.

19. Hirose T, Ohta S, Sato I, Matsuda M, Arai T, Katsura T. Tuberculous pleuro-peritonitis showing increased levels of CA-125. Nihon Kyobu Shikkan Gakkai Zasshi 1997; 35: 196-200.

20. Mas MR, Comert B, Saglamkaya U, Yamanel L, Kuzhan O, Ateskan U, et al. CA-125; a new marker for diagnosis and follow-up of patients with tuberculous peritonitis. Dig Liver Dis 2000; 32: 595-7.

21. Burgess LJ, Swanepoel CG, Taljaard JJ. The use of adenosine deaminase as a diagnostic tool for peritoneal tuberculosis. Tuberculosis (Edinb) 2001; 81: 243-8.

22. Fernández E, García S, Gutiérrez F, Ocio G, Rodrigo L, Riestra S. Diagnostic value of adenosine deaminase isoenzymes in ascitic fluid. AJG 1999; 94: 3658-60.

23. Hillebrand DJ, Runyon BA, Yasmineh WG, Rynders GP. Ascitic fluid adenosine deaminase insensitivity in detecting tuberculous peritonitis in the United States. Hepatology 1996; 24: 1408-12.

24. Hillebrand DJ, Runyon BA. Tuberculous peritonitis: Can ADA keep the laparoscope away?. Gastroenterology 1997; 113: 687-9.

25. Schwake L, von Herbay A, Junghanss T, Stremmel W, Mueller M. Peritoneal tuberculosis with negative polymerase chain reactions results: report of two cases. Scand J Gastroenterol 2003; 38: 221-4.

26. Hiller N, Lioubashevsky N. Tuberculous peritonitis : a diagnostic challenge. Abdom Imaging 2001; 26: 319-22.

27. Suri S, Gupta S, Suri R. Computed tomography in abdominal tuberculosis. Br J Radiol 1999; 72: 92-8.

28. Vázquez Muñoz E, Barbado Hernández FJ, Atienza Saura M. La tomografía computerizada en el diagnóstico de la peritonitis tuberculosa. Rev Clin Esp 2003; 203: 139-41. 